of LTR-CAT plasmids. The HTLV-2 $X$ product will act on HTLV-2 and HTLV-1 LTRs, whereas the $X$ product of HTLV-1 does not act on the HTLV-2 LTR. The promoter of HTLV-2 LTR is probably weaker than that of HTLV-1; this may explain its more restricted tropism to $\mathrm{T}$ cells, as only lymphoid cells will allow sufficient initial expression for the $\boldsymbol{X}$ gene product to act as a positive feedback. The long open reading frame in $X$ is highly conserved between HTLV-1 and HTLV-2 (ref.2).

The notion of trans-acting transcriptional activation may then be extrapolated to suggest that cellular control elements similar to LTRs might also be enhanced by the $X$ product. Interestingly, Holbrook $e t$ al. ${ }^{19}$ have just reported that a DNA sequence $5^{\prime}$ to the human interleukin 2 (IL-2) gene shows limited regions of homology to the LTR of HTLV-1. Since HTLV-transformed cells invariably express IL-2 receptors, activation of IL-2 by HTLV could lead to an autocrine stimulation of proliferation, possibly explaining the immortalization effect. But such a neat story is dashed by another recent paper ${ }^{20}$ showing that the IL-2 gene is not transcribed in HTLV-transformed cells. Perhaps expression of the IL-2 receptor gene itself is enhanced by the HTLV $X$ product.

The new observations on the apparent trans-acting regulation of HTLV expression will stimulate further exploitation of retroviruses and recombinant plasmids derived from them as probes for tissuespecific gene expression. It will be interesting to see whether the AIDS retrovirus isolates ${ }^{21,22}$, which destroy rather than immortalize OKT4 + cells, also have related $X$ genes and LTR enhancer sequences.

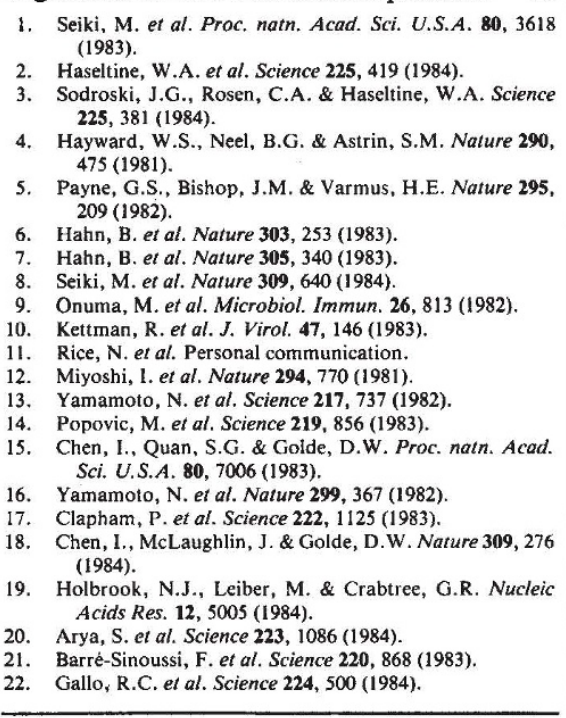

Robin Weiss is at the Institute of Cancer Research, Fulham Road, London SW3 6JB.

\title{
Oceanography
}

\section{Probing beneath the waves}

\section{from S.A. Thorpe}

A remarkable technical feat is described on page 282 of this issue of Nature. Because of the violence of the surface waves, precise measurement of the turbulence in the upper few tens of metres of the ocean is a daunting task. It demands great skill as well as stable instruments capable of high resolution, and few laboratories are adequately equipped. Nevertheless, Shay and Gregg at the University of Washington have now succeeded in using data from falling probes to show that in a convective layer below the surface of the ocean, a key property of the turbulence - the rate of dissipation of turbulence kinetic energy scales in the same way as it does in the convective boundary layer of the atmosphere ${ }^{l}$. This is not altogether a surprise. Others have suggested, and searched for, similarities between the ocean and atmospheric boundary layers ${ }^{2,3}$. It is, however, as well to be sure and to test the empirical relationships (empirical at present, but recently there have been theoretical advances ${ }^{4}$ ). Shay and Gregg provide a sounder basis for models which incorporate well-established information from the atmosphere. Their measurements also have practical implications, for example for estimating the distribution of nutrients which support phytoplankton, and for predicting the extreme currents which offshore structures must be able to withstand.

But is the upper ocean really like the lower atmosphere? What about the waves? There are strong indications that surface waves contribute significantly to ocean turbulence, at least close to the surface, probably through their breaking. The precise mechanism by which they do so is not known, although possible contributory factors include the generation of vorticity when the water surface becomes multiplyconnected in breaking waves, the detachment of the thin viscous boundary layer at the surface and motions in the wake of large rising bubbles of air engulfed by the breaking waves. There are basic asymmetries both in wave breaking and in the flows either side of the ocean-atmosphere interface. Thus, waves break at their crests, rather than at their troughs, and the falling wedge of water in a plunging breaker traps a bubble of air - an asymmetrical pattern; and the dynamically important level at which the mean fluid motion equals the speed of the dominant waves is located in the atmosphere rather than in the ocean. Therefore, whereas the non-wave-induced fluctuations dominate in the atmospheric surface layer, they are greatly exceeded by the wave-induced motions in the ocean surface layer. It is this which makes ocean tur- bulence so difficult to measure.

Evidence for the importance of waveinduced turbulence in the ocean surface layers is fragmentary and at the edge of confidence in experimental results. The most direct and convincing evidence comes from recent measurements of turbulence made from an instrumented tower in Lake Ontario. Kitaigorodski et al. ${ }^{5}$ found that the thickness of a surface layer in which wave-generated turbulence dominated that produced by other sources was of order ten times the root-mean-square wave amplitude. Indirect measurements based on the vertical distribution of bubbles below the surface ${ }^{6}$ support this result. What is astonishing is that such a thick layer should, on average, be affected, as wave-breaking is an intermittent and, when measured at a fixed position, rather infrequent event. It may be that another process ascribed to the action of surface waves is responsible for the downward advection of turbulence from the surface. In strong winds, parallel bands of foam or other flotsam aligned in the wind direction, and known as wind rows, appear on the sea surface. These are lines of convergence in alternating vortices known as Langmuir circulation $^{7}$ (after the man who first investigated the phenomena) and recent measurements show that the downward velocities beneath the wind rows may be several centimetres per second, sufficient to distort the distribution of turbulence and perhaps to thicken the layer appreciably.

Further surprises may be in store. Studies of drift currents ${ }^{8}$ show that the turbulent roughness of the surface is some two orders of magnitude greater than expected from atmospheric analogues. On this basis surface waves in moderate winds have a roughness scale for the water almost equal to that of a forest canopy for the air above.

Shay and Gregg have not measured the surface layer where wave-induced turbulence may dominate, but results may soon be available from a profiling instrument specially designed to profile upwards from depth to the surface in the wave zone ${ }^{9}$. We may confidently expect that, within the next few years, some of the intricate and fascinating connections between dynamical processes in the upperocean boundary layer will be unravelled so that models can be developed with more realistic representations of both local atmospheric forcing and waves.

1. Shay, T.J. \& Gregg, M.C. Nature 310, 282 (1984).

2. Jones, I.S. \& Kenney, B.C. J. geophys. Res. 82, 1392 (1977).

3. Dillon, T.M. et al, Nature 290,390 (1981).

4. Hunt, J.C.R. J. Fluid Mech. 138, 161 (1984)

S. Kitaigorodski, S.A., Donelan, M.A., Lumley, J.L. \& Terray,E.A. J. phys. Oceanogr. 13, 1988 (1983).

6. Thorpe, S.A. J. phys. Oceanogr. (in the press).

7. Leibovich, S. A. Rev. Fluid Mech. 15, 391 (1983)

8. Churchill, J.H. \& Csanady, G.T. J. phys. Oceanogr. 13, 1669 (1983).

9. Park, M.M. et al. WAZP Observations during MILDEX Oct-Nov 1983 (Sch. of Oceanogr., Oregon State Univ. 1984).

S. A. Thorpe is in the Institute of Oceanographic Sciences, Godalming, Surrey GU8 5 UB. 